\title{
3 Research Square

\section{Automatic Evaluation of Strephenopodia Based on Plantar Pressure and Machine Learning: A Pilot Study}

Jinjin Nong (D 591265029@qq.com )

South China University of Technology https://orcid.org/0000-0002-1854-9806

\section{Zikang Zhou}

SCUT: South China University of Technology

Xiaoming Xian

SCUT: South China University of Technology

Guowei Huang

SCUT: South China University of Technology

Peiwen Li

SCUT: South China University of Technology

Longhan Xie

SCUT: South China University of Technology

\section{Research Article}

Keywords: Strephenopodia assessment, Plantar pressure, Machine learning, Gait features

Posted Date: May 7th, 2021

DOI: https://doi.org/10.21203/rs.3.rs-434878/v1

License: (1) This work is licensed under a Creative Commons Attribution 4.0 International License.

Read Full License 


\title{
Automatic Evaluation of Strephenopodia Based on Plantar Pressure and Machine Learning: A Pilot Study
}

\author{
Jinjin Nong ${ }^{1} \cdot$ Zikang Zhou $^{1} \cdot$ Xiaoming Xian $^{1} \cdot$ Guowei Huang $^{1} \cdot$ Peiwen $\mathrm{Li}^{1} \cdot$ Longhan $\mathrm{Xie}^{1}$
}

\begin{abstract}
Purpose Stroke patients often suffer from strephenopodia because of high muscle tension or muscle spasms, which seriously affect their walking ability and rehabilitation. During the treatment of strephenopodia, there are practical demands for convenient, automatic, and quantitative assessments of the angle of strephenopodia. However, existing strephenopodia detection methods, including traditional clinical gait analysis, gait video analysis and plantar pressure systems, suffer from object obstruction or require complex setups. In this paper, we proposed a novel methodology for automatically predicting the angles of strephenopodia based on a gait analysis system using machine learning methods.

Methods Plantar pressure distribution data from thirty healthy participants were recorded during walking on the Zebris FDMTHM instrumented treadmill and were processed to generate 15 gait features. The right ankle angles on the coronal plane were measured by the Vicon system to provide a detailed description and explanation of strephenopodia walking. Three machine learning methods were implemented to build stochastic function mapping from gait features to strephenopodia angles.

Results This study showed good reliability and precision prediction of the angle of strephenopodia [determination coefficient $(\mathrm{R} 2) \geq 0.80]$. Gaussian process regression (GPR) exhibited the best regression performance [R2=0.93, mean root-mean-square error $(\mathrm{RMSE})=0.67]$.

Conclusion The study results showed that this strephenopodia-detection method is not only convenient to implement but also has high accuracy and outperforms previous reports. Measurements derived from the gait analysis system are proper estimators of the angle of strephenopodia and should be considered to improve diagnosis and assessment of the stroke population.
\end{abstract}

Keywords Strephenopodia assessment·Plantar pressure· Machine learning. Gait features

\begin{tabular}{l}
\hline Jinjin Nong and Zikang Zhou contributed equally to this work. \\
\hline Longhan Xie \\
melhxie@ scut.edu.cn \\
Jinjin Nong \\
$\underline{591265029 @ q q . c o m}$ \\
Zikang Zhou \\
mezikang@mail.scut.edu.cn \\
Xiaoming Xian \\
$\underline{769234221 @ q q . c o m}$ \\
Guowei Huang \\
$\underline{616126836 @ q q . c o m}$ \\
Peiwen Li \\
$\underline{\text { lintean@qq.com }}$
\end{tabular}

Shien-Ming Wu School of Intelligent Engineering, South China University of Technology, Guangzhou, 510640, China

\section{Introduction}

Stroke is one of the top leading causes of adult disability around the world [1], and $80 \%$ of the stroke patients tend to experience different degrees of walking obstacles [2]. Strephenopodia is one of the most common sequelae of stroke and is an adduction and supination varus posture. Strephenopodia seriously affects the patient's daily life and rehabilitation and may also cause serious injury [2-4]. To obtain better rehabilitation, it is necessary for patients to train themselves to walk properly using their own muscles [5]. If the muscles are not exercised because the foot rests on top of a comfortable orthotic shape, they would lose strength, and consequently, protective shock absorption, efficient gait [6] and other complex functions [7] become impaired. This highlights the necessity to automatically monitor the angle of strephenopodia to warn patients to consciously correct varus using their own muscles to achieve effective rehabilitation. 
Typical approaches for the detection of strephenopodia include traditional clinical gait analysis, gait video analysis and plantar pressure systems [5].

In the clinic, traditional gait analysis method [8] is mostly used for monitoring and providing feedback about strephenopodia before and after rehabilitation to evaluate treatment. Visual examination and standard goniometry are common tools in clinical assessment and diagnosis conducted by clinicians [4]. The physician observes the patient's gait features by visual inspection using clinical scale evaluations, such as the Brunnstrom assessment [9] and Clinical Spasticity Index (CSI) scores [10], and uses a measuring tool [5] to measure the angle of inversion. The results of this observational method rely on the observer's skill and clinical experience, and it cannot be widely used by nonprofessionals. In addition, concern for privacy leads to unnatural action when faced with the doctor's inquiries. Moreover, it is inconvenient to operate and cannot be recorded in real time in later stages. This method does not meet the requirements for clinical application $[11,12]$.

Gait video analysis is another method for strephenopodia evaluation. Computer vision [13], 3D kinematic analysis in vivo [4, 14], and optoelectronic stereophotogrammetry in vivo [15] were applied in patients with foot pathologies. Radiographic measurement [16] is also used for pronation and supination evaluation through the position of the talofirst metatarsal angle and the calcaneal-second and fifth metatarsal angles. However, this method is expensive and complex, which makes timely adjustment of the method inconvenient.

Since the acquisition of plantar pressure is simple and noninvasive, it has been widely used in detecting and monitoring gait patterns and lower extremity movements [17-19]. In a previous report [3], smart textile socks with five integrated pressure sensors were used for pronation and supination detection. Another study [5] utilized a pressuresensitive insole to detect foot pronation in real time, and feedback provided to the user reduced pronation significantly. Smart footwear insoles are implemented for recognition of foot pronation and supination other reports as well [20]. These methods are proven to be effective in detecting pronation and are relatively low cost. However, they are only able to detect whether foot pronation exists and cannot quantitatively determine the angle of foot pronation.

Therefore, it is critical to develop a simple, convenient, and noninvasive method to detect strephenopodia in clinical practice. The plantar pressure distribution can reflect lower limb movements $[18,19]$ in stroke survivors and serve as a strephenopodia-detection method. The electronic mats of Zebris FDM-THM instrumented treadmills are matrices of piezoresistive effect-based sensors. The Zebris treadmill can generate quantified values for gait and balance components, thus enabling exposure of subtle variations usually invisible to the naked eye [21, 22]. The "butterfly" diagram [21] from the instrumented treadmill reflects the variability of the center of the pressure trajectory during walking. Other parameters, such as the Center of Pressure (COP), double support phase (DSP), and cadence [23] for correlations and multiple classification analysis, were gathered with instrumented treadmills.

In this paper, we proposed a strephenopodia angle prediction method that can predict the foot supination angle based on the plantar pressure distribution. This method can be used to provide real-time feedback to the user to warn them about strephenopodia conditions. We collected a data set that consisted of plantar pressure distribution data and lower limb motion from 30 participants. Then, we fitted a Gaussian process regression (GPR) model to the data set. Experimental results indicate that our method can predict the foot supination angle with high precision. We also provide a performance comparison between the GPR and other regression models.

\section{Data collection and preprocessing}

Thirty healthy participants ( 25 males and 5 females) with no history of neurological or mobility impairments participated in the experiments. All participants provided informed consent, and the experimental procedures were approved by the Research Ethics Board of South China University of Technology. Table 1 provides summary information about the participants.

Table 1 The distribution of participants' body parameters

\begin{tabular}{cc}
\hline Characteristics & Mean \pm SD \\
\hline Age (years) & $22.83 \pm 2.83$ \\
Height $(\mathrm{cm})$ & $169.81 \pm 7.38$ \\
Weight $(\mathrm{kg})$ & $64.21 \pm 12.54$ \\
\hline
\end{tabular}

\subsection{Experimental Setup}

The experimental setup included three parts: a Zebris FDM-THM Treadmill (Zebris Medical GmbH, Germany) for measuring the plantar pressure distribution, wedge- 
shaped blocks for simulating strephenopodia conditions, and a Vicon Motion Capture System for capturing lower limb motion.

Platform systems embedded in a treadmill are considered the gold standard in plantar pressure measurement and have high measurement precision [24]. We utilized the Zebris FDM-THM Treadmill (Figure 1) to obtain the gait features.

We designed three wedge-shaped blocks with different heights. In the experiment, the blocks were stuck under the forefoot to simulate different strephenopodia conditions.

\section{A: Whole view}

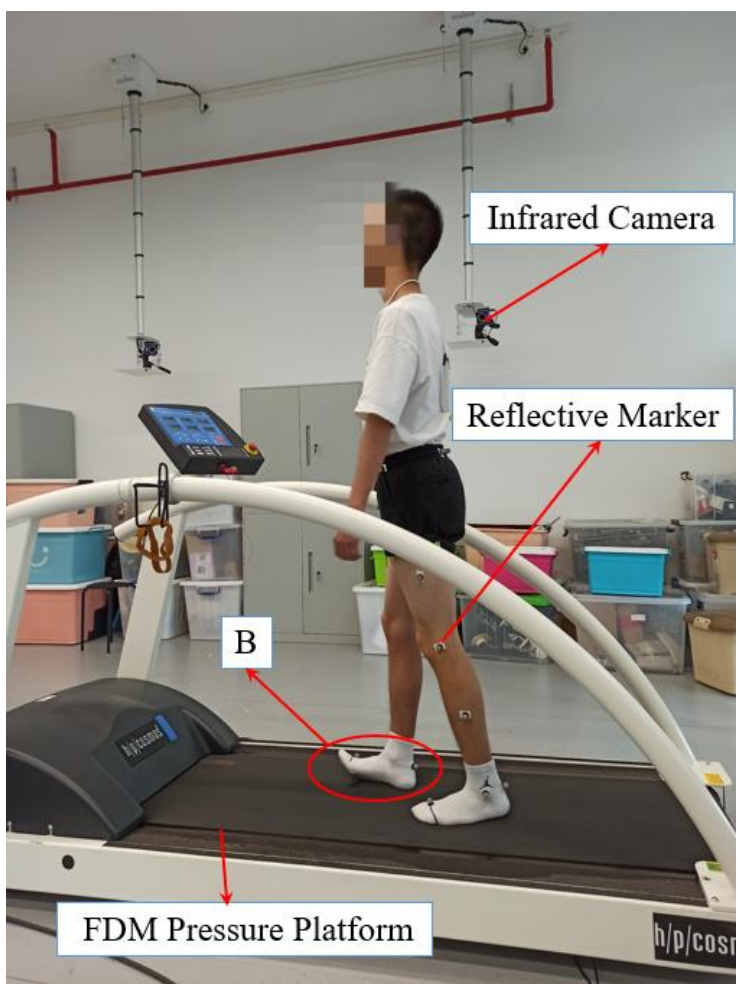

B: Partial schematic

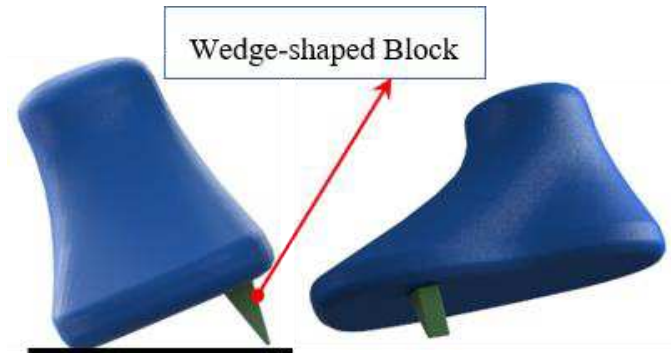

Fig. 1 Experimental setup with the Zebris FDM-THM Treadmill and Vicon Motion Capture System. The sampling rate of Zebris Treadmill is set to $120 \mathrm{~Hz}$. The Vicon Motion Capture System consists of 8 infrared cameras, the sampling rate is set to $250 \mathrm{~Hz}$. The cameras capture the movement of markers that are used to reconstruct a lower limb model for calculating ankle angles. The wedge-shaped blocks are stuck to the first metatarsal area to form a specific angle to imitate varus conditions.
The higher the block, the more severe the simulated foot supination. Participants walked at self-selected speeds on the treadmill under the 4 different conditions. In the first condition, the participants walked freely with no block; then, for the other three conditions, blocks with different heights were stuck to the first metatarsal area of the medial forefoot of the right foot. We asked the participants to keep the blocks close to the ground as much as possible but not touch the treadmill belt to ensure a certain angle when walking, which imitates the different degrees of strephenopodia conditions.

We attached 16 reflective markers to the subject, and details about the marker positions are described in reference [25]. The Vicon system measures the trajectories of these 16 reflective markers, which will be used to calculate joint kinematics later. Gait patterns are represented by lower limb joint trajectories: ankle adduction/abduction on the coronal plane, mainly referring to strephenopodia, and other joint rotations are not considered.

\subsection{Measurement Procedure}

First, we asked all participants to participate in an adaptation-familiarization trial to obtain a self-selected walking speed and adapt and maintain the inversion angle. Starting at a fixed speed of $0.5 \mathrm{~km} / \mathrm{h}$, the treadmill speed was increased by $0.2 \mathrm{~km} / \mathrm{h}$ every $10 \mathrm{~s}$ in a stepwise manner. Once the participant informed the tester of the speed that best characterized his/her normal walking pace, that was determined as his/her comfortable speed. After this adaptation phase, each participant was asked to walk continuously for approximately 2 minutes on a treadmill at his/her comfortable speed for each walking model while wearing socks. When a stable walking speed was reached, motion capture data were recorded synchronously with the treadmill for 30 seconds. Gait assessment was performed in the laboratory to ensure a quiet environment.

All subjects performed four types of walking motions with their right foot. Each walking model recorded 8 groups of data, 30 seconds per group. In addition, each subject was asked to simulate common post stroke strephenopodia movements, which included the flexion of the affected toe, the anterolateral edge of the plantar, and the inclination angle between the plantar and the ground. Then, the raw data from Vicon and the treadmill are synchronously recorded for regressions to predict the degree of strephenopodia. 


\subsection{Preprocessing of Joint Trajectories}

All the raw gait trajectories obtained by the Vicon system need to go through a series of processes. 1) We removed invalid gait patterns caused by marker occlusions. 2) We applied low-pass filtering using a Butterworth low-pass filter with a cutoff frequency of $6 \mathrm{~Hz}$ to reduce noise in raw signals. Figure 2 shows the filtered right ankle angle trajectory. 3) The gait sequence was split into gait cycles to obtain the average of the minimum value of each gait cycle for ankle flexion/extension because we found that in the support phase, the varus foot was relatively smaller throughout the gait cycle. 4) The average of the minimum right ankle value of each gait cycle was calculated,

$$
y_{i}=\frac{1}{n} \sum_{j=1}^{n} y_{j},
$$

where $n$ is the number of gait cycles, $y_{j}$ is the minimum value of the right ankle angle throughout the $j$-th gait cycle, and $y_{i}$ is considered to be the strephenopodia angle.

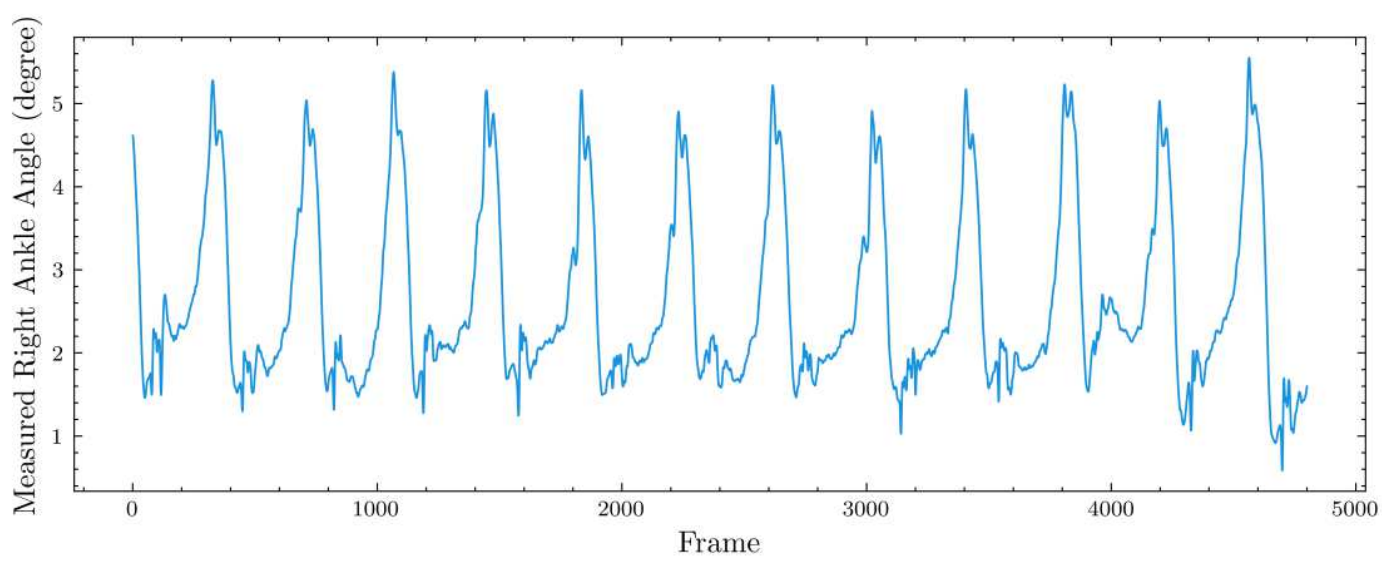

Fig. 2 Right ankle angles on the coronal plane.

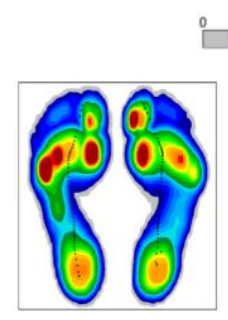

(a)

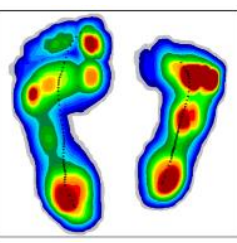

(b)

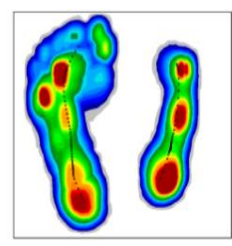

(c)

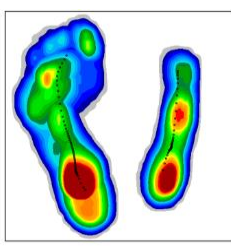

(d)

Fig. 3 The plantar pressure distribution in the stance phase. (a) Healthy gait; the degree of strephenopodia increases from (b) to (d).

Table 2 Descriptions of gait features from the Zebris gait analysis system

\begin{tabular}{|c|c|}
\hline Gait feature & Description \\
\hline Fore foot force & the ratio of maximum force in the fore foot to the body weight \\
\hline Loading response phase & the phase between the initial ground contact and contralateral toe off \\
\hline Length of the gait line & the gait characterized by the position of the center of pressure (COP) \\
\hline Gait line left and right & the lines of the force application points shown separately for each foot \\
\hline Anterior/Posterior Position & the shift forwards/backwards of the COP intersection point in chronological sequence in the cyclogram display \\
\hline Lateral shift & the left/right shift of the COP intersection point in chronological sequence in the cyclogram display \\
\hline Load change & the absolute load change from the heel to the forefoot during the stance phase given as a percentage \\
\hline $\begin{array}{l}\text { Contact time, percentage of } \\
\text { stance time }\end{array}$ & the average contact time of the three zones, toes, mid-foot and heel as a percentage. \\
\hline Stance phase & the phase of a gait cycle in which the foot has contact with the ground. \\
\hline 3 Maximum force & the average maximum values reached in $\mathrm{N}$ for the three zones: toes, mid-foot and heel. \\
\hline 3 Maximum pressure & the average maximum values reached in $\mathrm{N} / \mathrm{cm}^{2}$ for the three zones: toes, mid-foot and heel. \\
\hline
\end{tabular}




\subsection{Gait Feature Extraction}

As shown in Figure 3, the plantar pressure distribution is a good description of the different severities of strephenopodia. The data dimensionality of plantar pressure is too large to be directly applied in machine learning algorithms. Therefore, we extracted 15 gait features from the raw plantar pressure data collected by the Zebris gait analysis system (Table 2).



(a)

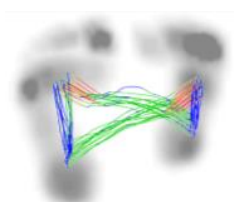

(b)

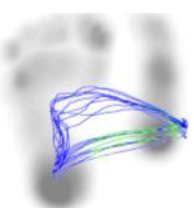

(c)
Fig. 4 The "butterfly" diagrams derived from the Zebris treadmill: (a) Normal gait. (b) Gait with mild pronation. (c) Gait with severe pronation.

"Butterfly" diagrams are presented in Figure 4. There are obvious differences between both foot asymmetries in gait phases that can be obtained [2] and have more asymmetrical stance times compared with healthy subjects. The shadow of the right footprint also decreased, indicating varus deepening in the foot.

These 15 gait features were extracted from the raw plantar pressure data and served as the input features of our dataset to train the different regression models.

\section{Prediction of strephenopodia angles}

We implemented three regression models Gaussian process regression (GPR), Support vector regression (SVR), and Stepwise linear regression (SLR), to evaluate their performance in predicting strephenopodia angles from gait features.

\subsection{Definition of Training Set}

The training set consists of the input set $\boldsymbol{X}$ and the output set $\boldsymbol{Y}$. The input set $\boldsymbol{X}$ consists of vectors of gait features, and the output set $\boldsymbol{Y}$ consists of output scalars (strephenopodia angles):

$$
\boldsymbol{X}=\left[\begin{array}{c}
\boldsymbol{x}_{\mathbf{1}}^{T} \\
\vdots \\
\boldsymbol{x}_{N}^{T}
\end{array}\right], \boldsymbol{Y}=\left[\begin{array}{c}
y_{1} \\
\vdots \\
y_{N}
\end{array}\right],
$$

where $\mathrm{N}$ is the number of samples in the data set (specifically 379).
The regression models will be optimized to build mapping relationships between the input $X$ and the output $Y$.

\subsection{Gaussian Process Regression}

A Gaussian process (GP) [26-28] is a stochastic process specified by its mean function and its covariance function, $m(x), k\left(x, x^{\prime}\right)$ :

$$
f(x) \sim G P\left(m(x), k\left(x, x^{\prime}\right)\right) .
$$

In our work, the mean function and covariance function are determined as follows:

$$
\begin{gathered}
m(x)=0, \\
k\left(x, x^{\prime}\right)=\sigma_{f}^{2}\left[1+\frac{\left(x^{i}-x^{j}\right)^{T} M\left(x^{i}-x^{j}\right)}{2 \alpha}\right]^{-\alpha},
\end{gathered}
$$

where $\sigma_{f}^{2}$ is the signal variance of the kernel function, $\alpha$ is the shape parameter of the kernel function, and $M=$ $\operatorname{diag}\left(l^{-2}\right)$ is a symmetric matrix of hyperparameters.

With a given test set $x^{*}$, we can predict a probability distribution of ankle angle $y^{*}$ based on the above configuration:

$$
p\left(y^{*} \mid \mathbf{x}^{*}, \mathbf{X}, \mathbf{y}, \Theta\right)=\mathcal{N}\left(\mathbf{k}_{*}^{\top} \mathbf{K}^{-1} \mathbf{y}, \kappa-\mathbf{k}_{*}^{\top} \mathbf{K}^{-1} \mathbf{k}_{*}\right),
$$

where $K$ is the covariance matrix with elements $K_{i j}=$ $k\left(x_{i}, x_{j}\right) . k^{*}=\left[k\left(x^{*}, x_{x}\right) \ldots k\left(x^{*}, x_{n}\right)\right]^{T} . \kappa=k\left(x^{*}, x^{*}\right)$.

\subsection{Support Vector Regression}

The support vector regression (SVR) model is characterized by its kernels, sparse solution, and VapnikChervonenkis (VC) control of the margin and the number of support vectors. SVR models are trained using a symmetrical loss function, which equally penalizes high and low misestimates. In $\varepsilon$-SV regression [29-32], our goal is to find a function, $f(x)$, that has at most $\varepsilon$ deviation from the actually obtained targets $y_{i}$ for all the training data and is as flat as possible. We construct a linear regression function:

$$
f(x)=W^{T} \Phi(x)+b,
$$

where $W$ and $b$ are obtained by solving an optimization problem:

$$
\begin{gathered}
\min _{W, b} P=\frac{1}{2} W^{T} W+C \sum_{i=1}^{n}\left(\xi_{i}+\xi_{i}^{*}\right), \\
\text { s.t. }\left\{\begin{array}{c}
y_{i}-\left(W^{T} \Phi(x)+b\right) \leq \varepsilon+\xi_{i}, \\
\left(W^{T} \Phi(x)+b\right)-y_{i} \leq \varepsilon+\xi_{i}^{*}, \\
\xi_{i}, \xi_{i}^{*} \geq 0, \quad i=1 \cdots n .
\end{array}\right.
\end{gathered}
$$


The optimization criterion penalizes data points whose yvalues differ from $f(x)$ by more than $\varepsilon$. The slack variables, $\xi$ and $\xi^{*}$, correspond to the size of this excess deviation for positive and negative deviations.

\subsection{Stepwise Linear Regression}

Stepwise linear regression [33-35] is a multivariate statistical data analysis method that studies the correlation between dependent variables and multiple influencing factors and is widely used in prediction and control. In the preliminary regression analysis, we first establish the total regression equation between the dependent variable $y$ and the independent variable $\mathrm{x}$ and then perform hypothesis testing on the total equation and each independent variable. If the regression equation reaches a satisfactory level, the algorithm terminates. Otherwise, some variables that are not significantly different will be eliminated using the interpreted information. This process is an iterative process to ensure that the final set of explanatory variables is optimal.

\section{Results}

The methods are tested with the five-fold cross-validation method. We randomly divide the collected data set into five groups. For each of the five iterations, four groups are used as the training set, and the other group is used as the test set. The hyperparameters of these three algorithms are optimized by the grid search method. The results are aggregated to verify the models to find the average prediction accuracy of the system [36].

We selected the root-mean-square error (RMSE) and determination coefficient (R2) as model evaluation parameters. The correlation index R2 describes the fit of the regression model. The closer $\mathrm{R} 2$ is to 1 , the better the fit of the model. The R2 for individual regressors reached 1, indicating that the models exhibited the desired prediction performance. R2 and RMSE were formulated as follows:

$$
\begin{gathered}
\text { RMSE }=\sqrt{\frac{1}{n} \sum_{i=1}^{n}\left(y_{i}-\hat{y}_{i}\right)^{2},} \\
R^{2}=1-\frac{\sum_{i=1}^{n}\left(y_{i}-\hat{y}_{i}\right)^{2}}{\sum_{i=1}^{n}\left(y_{i}-\bar{y}\right)^{2}},
\end{gathered}
$$

where $n$ is the number of samples, $y_{i}$ is the right ankle angle, $\bar{y}$ is the mean value of the right ankle angle and $\hat{y}_{i}$ is the predicted angle.

The regression accuracies for the three machine learning regression methods are shown in Table 3 and Figure 5. The
Table 3 Regression performance

\begin{tabular}{ccc}
\hline Method & R2 \pm SD & RMES \pm SD \\
\hline GPR & $0.93 \pm 0.01$ & $0.67 \pm 0.03$ \\
SVR & $0.83 \pm 0.04$ & $1.06 \pm 0.14$ \\
SLR & $0.80 \pm 0.04$ & $1.13 \pm 0.15$ \\
\hline
\end{tabular}
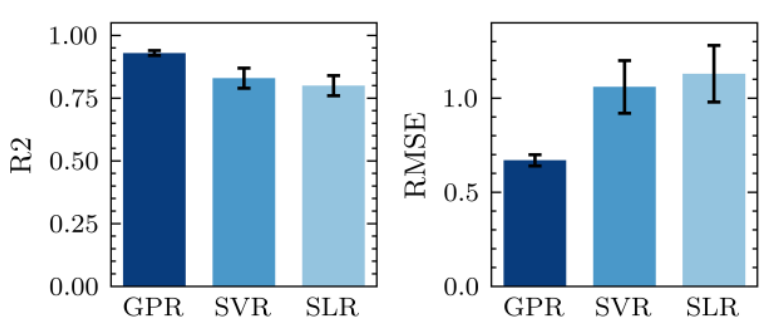

Fig. 5 R2 and RMSE metrics for the GPR, SVR and SLR.

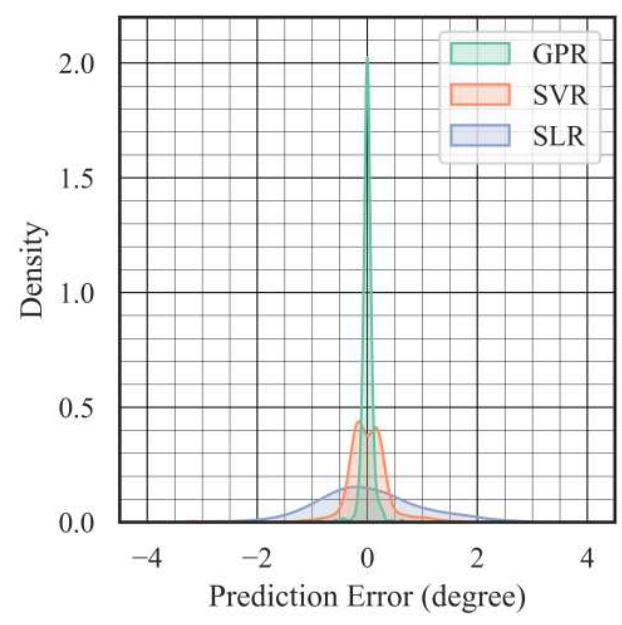

Fig. 6 Kernel density estimate (KDE) plots for the prediction errors of three regression models. The distributions of the prediction errors are demonstrated intuitively in these plots.

GPR method achieved the best prediction performance, with the highest R2 (0.93) and lowest RSME (0.67) among all regression algorithms. All regression algorithms had a higher R2 ( $\geq 0.80)$ in the walking tasks. These algorithms all showed relatively good ability to predict the angle.

Figure 6 shows the kernel density estimate (KDE) plots for the prediction errors of the three regression models. The prediction error of the GPR method basically falls within the range of -0.5 to 0.5 , showing that the GPR method achieved excellent prediction.

Figure 7 shows the prediction results of the regression algorithms in a more intuitive way. Actual right ankle angles obtained from the Vicon system are compared with the prediction results from three regression models. The 

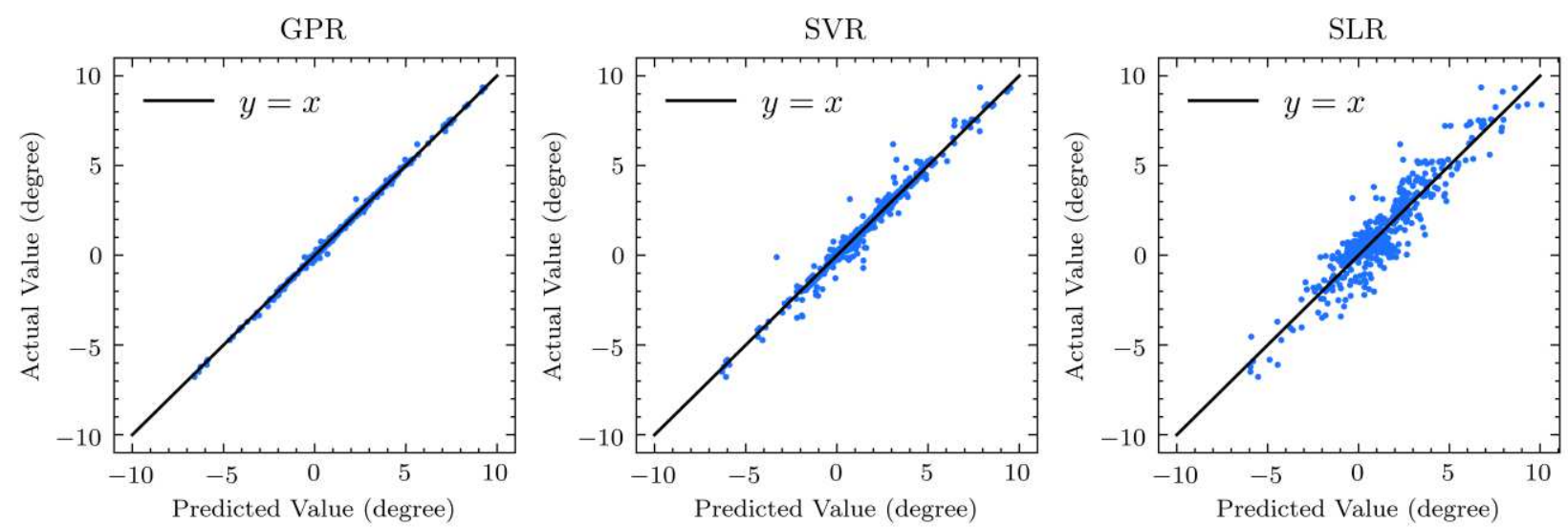

Fig. 7 Actual angle and predicted angle of three regression models.

predicted results of the three models are in relatively good agreement with the actual values, and the predicted results meet the accuracy requirements.

In summary, the GPR showed the best performance in predicting strephenopodia angles from gait features, SLR had the worst predictive performance, and SVR was somewhere in between.

\section{Discussion and conclusions}

Currently, plantar pressure systems are usually used for the recognition of foot pronation and supination and do not detect the angle of varus in the foot. Compared to the detection of strephenopodia from a scale evaluation, our method provides a more convenient, quantitative and automatic method of detecting varus based on a gait pressure distribution system.

The major factor limiting our prediction accuracy is the subject-specific difference in gait characteristics. Because of the differences in their age, gender, weight, leg length and other factors, different participants tended to exhibit unique gait characteristics. These subject-specific differences are more significant than pattern-specific differences caused by different strephenopodia conditions. One solution to this problem is to enlarge the size of the training data by increasing the number of participants. Another factor is that walking may involve randomness [37], which can cause prediction error. Sometimes it would cause an unstable center of gravity or a sudden disorder of pace. Some steps in most figures have significantly different pressure vector shapes, which is explained as missing steps that participants reported making occasionally [3].

Our method has a number of advantages. First, it is noninvasive, and measuring plantar pressure does not induce unnatural movements caused by attached sensors or negative feelings caused by being monitored. Second, the pressure distribution-based strephenopodia detection method is objective, quantitative and more practical than scale evaluation methods or camera-based methods. Third, it does not require complex equipment. A pressure treadmill is sufficient and can even be reduced to a pressure insole. There is no need for complex equipment, such as motion capture systems that require complicated setups.

The major limitation of our work is that our regression models are only tested on simulated data from healthy participants instead of data acquired from stroke survivors. Although common strephenopodia walking movement patterns were simulated by healthy participants in this paper, strephenopodia walking patterns may be more variable in stroke survivors. Future research is needed to explore the regression performance in predicting the strephenopodia angles of stroke survivors.

In this paper, we proposed and tested the use of plantar pressure distribution for predicting strephenopodia angles. This novel method can provide quantitative and unobtrusive monitoring of strephenopodia conditions. We extracted 15 gait features from the plantar pressure data and then used three different regression algorithms to establish mapping relationships between the gait features and the strephenopodia angle. Systematic experimental results have shown that the GPR algorithm achieved excellent performance $(\mathrm{R} 2=0.93, \mathrm{RMSE}=0.67)$ in strephenopodia angle prediction based on gait features obtained from plantar pressure.

Acknowledgments We are grateful to Ms. Xiaoya Zhang, for providing some professional knowledge and valuable 
comments in this study from The Third Affiliated Hospital, Sun Yat-sen University, Guangzhou 510630, China.

Funding This work was supported in part by the National Natural Science Foundation of China (Grant No. 52075177), the Joint Fund of the Ministry of Education for Equipment Pre-Research (Grant No. 6141A02033124), the Research Foundation of Guangdong Province (Grant No. 2019A050505001 and 2018KZDXM002), the Guangzhou Research Foundation (Grant No. 202002030324 and 201903010028), the Zhongshan Research Foundation (Grant No.2020B2020), and the Shenzhen Institute of Artificial Intelligence and Robotics for Society (Grant No. AC01202005011).

\section{Compliance with Ethical Standards}

Conflict of interest None.

Ethical Approval Yes.

Consent to Participate Yes.

\section{References}

1. Burton, J. K., Ferguson, E. E. C., Barugh, A. J., Walesby, K. E., MacLullich, A. M. J., Shenkin, S. D., \& Quinn, T. J. (2018). Predicting Discharge to Institutional Long-Term Care After Stroke: A Systematic Review and Metaanalysis. Journal of the American Geriatrics Society, 66(1), 161-169. https://doi.org/10.1111/jgs.15101

2. Qiu, S., Wang, Z., Zhao, H., Liu, L., \& Jiang, Y. (2018). Using Body-Worn Sensors for Preliminary Rehabilitation Assessment in Stroke Victims With Gait Impairment. IEEE Access, 6, 31249-31258. https://doi.org/10.1109/ACCESS.2018.2816816

3. Eizentals, P., Katashev, A., Okss, A., Pavare, Z., \& Balcuna, D. (2019). Detection of Excessive Pronation and Supination for Walking and Running Gait with Smart Socks. In L. Lhotska, L. Sukupova, I. Lacković, \& G. S. Ibbott (Eds.), World Congress on Medical Physics and Biomedical Engineering 2018 (Vol. 68/2, pp. 603-607). Singapore: Springer Singapore. https://doi.org/10.1007/978-981-10-90387_112

4. Alonso-Vázquez, A., Villarroya, M. A., Franco, M. A., Asín, J., \& Calvo, B. (2009). Kinematic assessment of paediatric forefoot varus. Gait \& Posture, 29(2), 214-219. https://doi.org/10.1016/j.gaitpost.2008.08.009
5. Berengueres, J., Fritschi, M., \& McClanahan, R. (2014). A smart pressure-sensitive insole that reminds you to walk correctly: An orthotic-less treatment for over pronation. In 2014 36th Annual International Conference of the IEEE Engineering in Medicine and Biology Society (pp. 2488-2491). Presented at the 2014 36th Annual International Conference of the IEEE Engineering in Medicine and Biology Society (EMBC), Chicago, IL: IEEE. https://doi.org/10.1109/EMBC.2014.6944127

6. Ker, R. F., Bennett, M. B., Bibby, S. R., Kester, R. C., \& Alexander, R. McN. (1987). The spring in the arch of the human foot. Nature, 325(6100), 147-149. https://doi.org/10.1038/325147a0

7. Kelly, L. A., Cresswell, A. G., Racinais, S., Whiteley, R., \& Lichtwark, G. (2014). Intrinsic foot muscles have the capacity to control deformation of the longitudinal arch. Journal of The Royal Society Interface, 11(93), 20131188. https://doi.org/10.1098/rsif.2013.1188

8. Fopma, E., Abboud, R., \& Macnicol, M. (2003). CORRELATION OF A CLINICAL OUTCOME MEASUREMENT TO BIO-MECHANICAL ASSESSMENT IN SURGICALLY TREATED CLUBFEET. In Orthopaedic Proceedings (Vol. 85, pp. 168-168). The British Editorial Society of Bone $\&$ Joint Surgery.

9. Guo-Wei, W. U. (2007). Observation on Therapeutic Effect of Neuromuscular Facilitation Techniques in the Strephenopodia after Stroke. Practical Clinical Medicine.

10. Yong, Z., Li-Xin, F. U., Feng, W., Gui-Quan, C., Hai-Feng, L. I., \& Yuan-Hui, D. U. (2016). Systematic Evaluation of Acupuncture for the Treatment of Post-stroke Strephenopodia. Journal of Clinical Acupuncture and Moxibustion.

11. Zhao, W., Reinthal, M. A., Espy, D. D., \& Luo, X. (2017). Rule-Based Human Motion Tracking for Rehabilitation Exercises: Realtime Assessment, Feedback, and Guidance. IEEE Access, 5, 21382 21394. https://doi.org/10.1109/ACCESS.2017.2759801

12. Taborri, J., Palermo, E., Rossi, S., \& Cappa, P. (2016). Gait Partitioning Methods: A Systematic Review. Sensors, 16(1), 66. https://doi.org/10.3390/s16010066 
13. Pecar, A. (2014). Automatic detection of pronation and supination of runners using computer vision.

14. Davis, I. S. (2004). Measuring Foot Motion: Forward and Inverse Dynamic Models-Foot and Ankle Research Retreat II, April 30-May 1, 2004, Los Angeles, California. Journal of Orthopaedic \& Sports Physical Therapy, 34(9).

15. Leardini, A., Benedetti, M. G., Berti, L., Bettinelli, D., Nativo, R., \& Giannini, S. (2007). Rear-foot, mid-foot and fore-foot motion during the stance phase of gait. Gait \& Posture, 25(3), 453-462. https://doi.org/10.1016/j.gaitpost.2006.05.017

16. Fridman, M. W., \& de Almeida Fialho, H. S. (2007). The role of radiographic measurements in the evaluation of congenital clubfoot surgical results.

Skeletal Radiology, 36(2), 129-138. https://doi.org/10.1007/s00256-006-0204-3

17. Lee, S.-S., Choi, S. T., \& Choi, S.-I. (2019). Classification of Gait Type Based on Deep Learning Using Various Sensors with Smart Insole. Sensors, 19(8), 1757. https://doi.org/10.3390/s19081757

18. Buldt, A. K., Forghany, S., Landorf, K. B., Levinger, P., Murley, G. S., \& Menz, H. B. (2018). Foot posture is associated with plantar pressure during gait: a comparison of normal, planus and cavus feet. Gait \& Posture, 62, 235.

19. Buldt, A. K., Allan, J. J., Landorf, K. B., \& Menz, H. B. (2018). The relationship between foot posture and plantar pressure during walking in adults: A systematic review. Gait \& Posture, 56.

20. Domínguez-Morales, M. J., Luna-Perejón, F., MiróAmarante, L., Hernández-Velázquez, M., \& Sevillano-Ramos, J. L. (2019). Smart Footwear Insole for Recognition of Foot Pronation and Supination Using Neural Networks. Applied Sciences, 9(19), 3970. https://doi.org/10.3390/app9193970

21. Kalron, A., \& Frid, L. (2015). The "butterfly diagram": A gait marker for neurological and cerebellar impairment in people with multiple sclerosis. Journal of the Neurological Sciences, 358(1-2), 92-100. https://doi.org/10.1016/j.jns.2015.08.028

22. Chen, S.-H. (Teresa), Taylor, J., \& Chou, L.-S. (2017). Lower Limb Muscle Fatigue Perturbs Gait Balance during Dual-Task Walking: 2437 June 29 30 AM - 945 AM. Medicine \& Science in Sports \&
Exercise, 49(5S), 683.

https://doi.org/10.1249/01.mss.0000518805.82817.e7

23. Fuchioka, S., Iwata, A., Higuchi, Y., Miyake, M., Kanda, S., \& Nishiyama, T. (2015). The Forward Velocity of the Center of Pressure in the Midfoot is a Major Predictor of Gait Speed in Older Adults. International Journal of Gerontology, 9(2), 119-122. https://doi.org/10.1016/j.ijge.2015.05.010

24. Drăgulinescu, A., Drăgulinescu, A.-M., Zincă, G., Bucur, D., Feieș, V., \& Neagu, D.-M. (2020). Smart Socks and In-Shoe Systems: State-of-the-Art for Two Popular Technologies for Foot Motion Analysis, Sports, and Medical Applications. Sensors, 20(15), 4316. https://doi.org/10.3390/s20154316

25. Vicon Motion Systems Limited. (2016). Plug-in Gait Reference Guide.

26. Rasmussen, C. E., \& Williams, C. K. I. (2006). Gaussian Processes for Machine Learning. MIT Press.

27. Ebden, M. (2015). Gaussian Processes for Regression and Classification: A Quick Introduction. Statistics.

28. Lizotte, D. J., Wang, T., Bowling, M. H., \& Schuurmans, D. (2007). Automatic Gait Optimization with Gaussian Process Regression. In International Joint Conference on Artifical Intelligence.

29. Cortes, C., \& Vapnik, V. N. (1995). Support-Vector Networks. Machine Learning, 20(3), 273-297.

30. Smola, A. J., \& Schölkopf, B. (2004). A tutorial on support vector regression. Statistics and Computing, 14(3), 199-222.

https://doi.org/10.1023/B:STCO.0000035301.49549. 88

31. Ma, J., Theiler, J., \& Perkins, S. (2003). Accurate On-line Support Vector Regression. Neural Computation, 15(11), 2683-2703. https://doi.org/10.1162/089976603322385117

32. Hawkins, J., \& BODÉN, M. (2008). DETECTING AND SORTING TARGETING PEPTIDES WITH NEURAL NETWORKS AND SUPPORT VECTOR MACHINES. Journal of Bioinformatics and Computational Biology, 04(01), 1-18.

33. Yuekai, C. (2012). Stepwise Linear Regression Prediction Model for Civil Vehicle Quantity of China. Journal of Heb Jiaotong Vocational and Technical College. 
34. Fan-Jie, L., \& Dan-Lu, S. (2015). Optimization of the Temperature Measuring Points Based on Linear Stepwise Regression. Modular Machine Tool \& Automatic Manufacturing Technique.

35. Malyuto, V., \& Shvelidze, T. (1989). The technique of automatic quantitative stellar spectral classification using stepwise linear regression. Astrophysics and Space Science, 155(1), 71-83. https://doi.org/10.1007/BF00645208

36. Kohavi, R. (1995). A study of cross-validation and bootstrap for accuracy estimation and model selection. In International joint conference on Artificial intelligence.

37. Hausdorff, J. M., Peng, C. K., Ladin, Z., Wei, J. Y., \& Goldberger, A. L. (1995). Is walking a random walk? Evidence for long-range correlations in stride interval of human gait. Journal of Applied Physiology, 78(1), 349-358. 


\section{Figures}

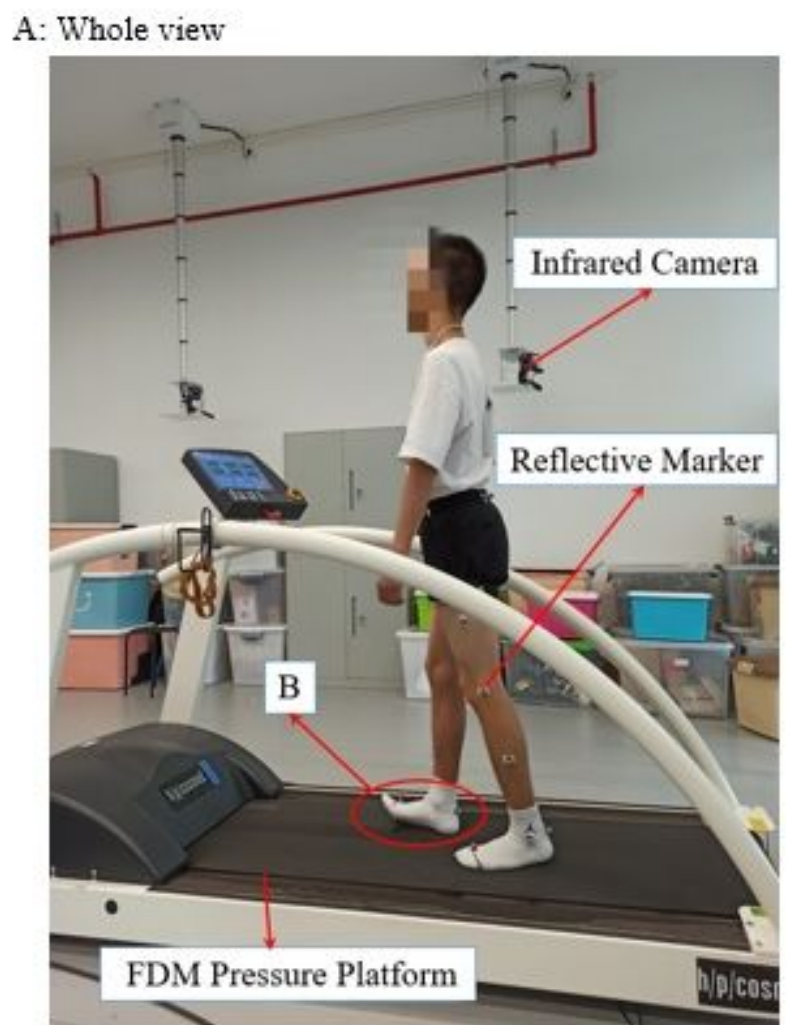

B: Partial schematic

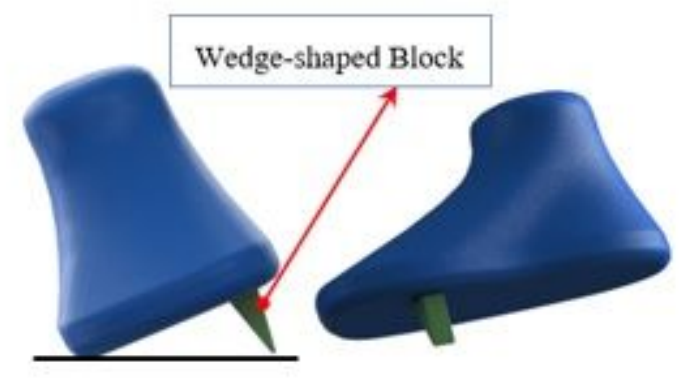

\section{Figure 1}

Experimental setup with the Zebris FDM-THM Treadmill and Vicon Motion Capture System. The sampling rate of Zebris Treadmill is set to $120 \mathrm{~Hz}$. The Vicon Motion Capture System consists of 8 infrared cameras, the sampling rate is set to $250 \mathrm{~Hz}$. The cameras capture the movement of markers that are used to reconstruct a lower limb model for calculating ankle angles. The wedge-shaped blocks are stuck to the first metatarsal area to form a specific angle to imitate varus conditions. 


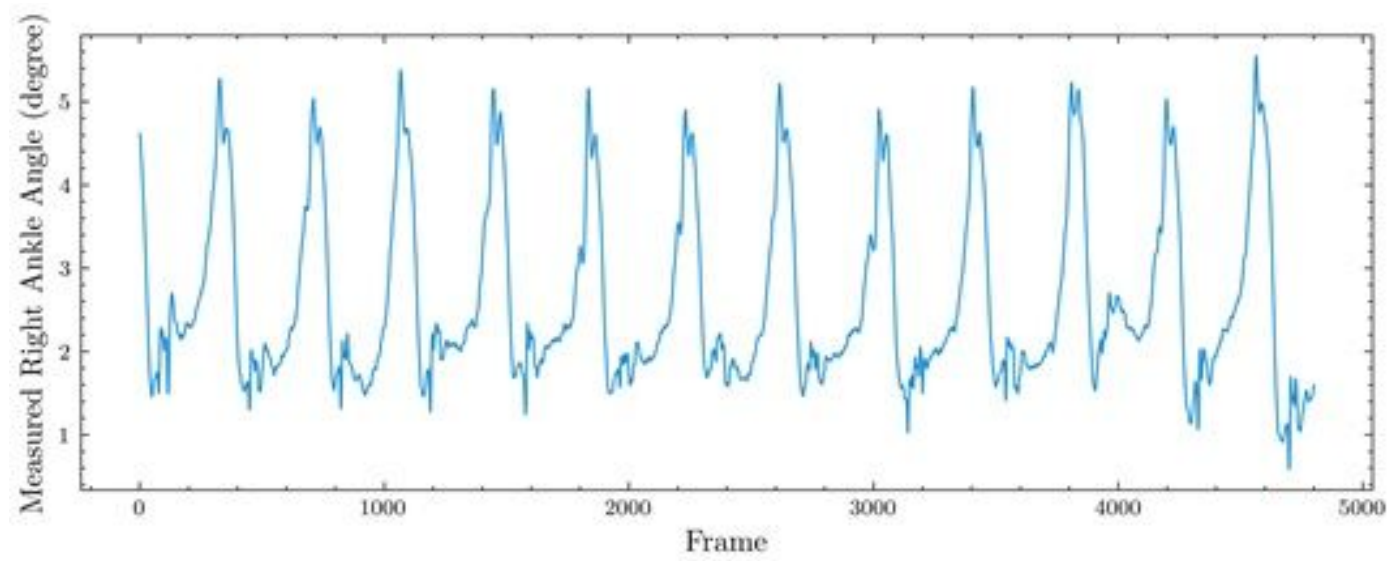

Figure 2

Right ankle angles on the coronal plane.

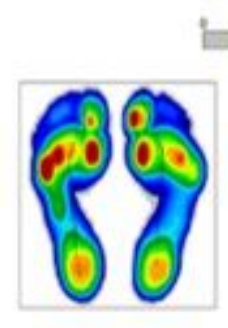

(a)

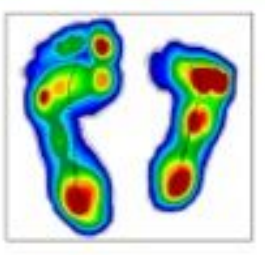

(b)

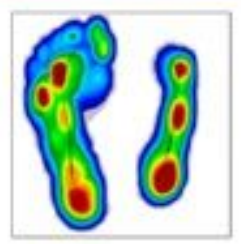

(c)

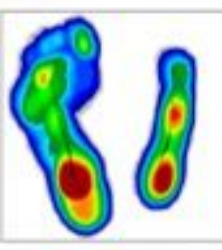

(d)

\section{Figure 3}

The plantar pressure distribution in the stance phase. (a) Healthy gait; the degree of strephenopodia increases from (b) to (d).

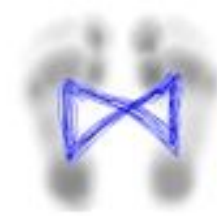

(a)



(b)

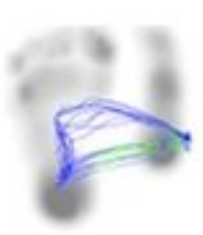

(c)

Figure 4

The "butterfly" diagrams derived from the Zebris treadmill: (a) Normal gait. (b) Gait with mild pronation. (c) Gait with severe pronation.
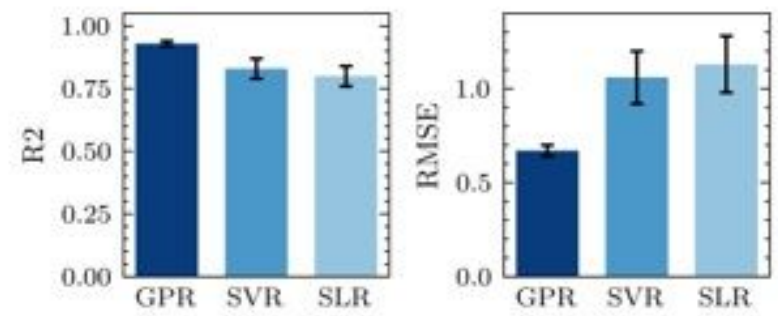
Figure 5

R2 and RMSE metrics for the GPR, SVR and SLR.

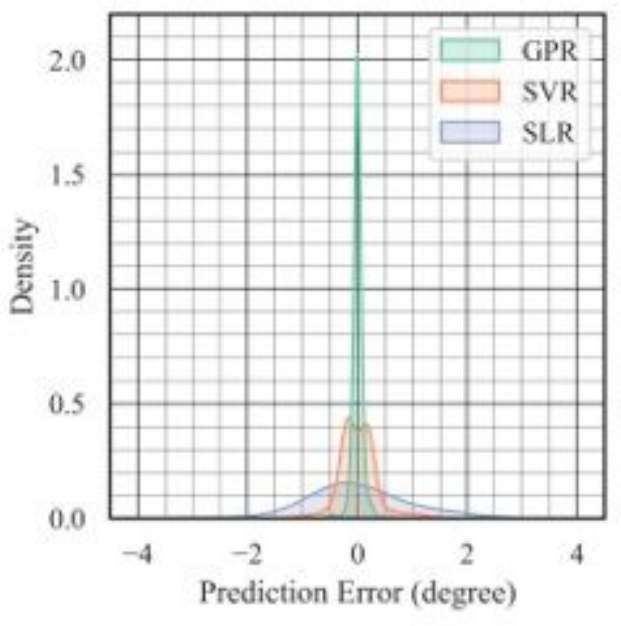

Figure 6

Kernel density estimate (KDE) plots for the prediction errors of three regression models. The distributions of the prediction errors are demonstrated intuitively in these plots.
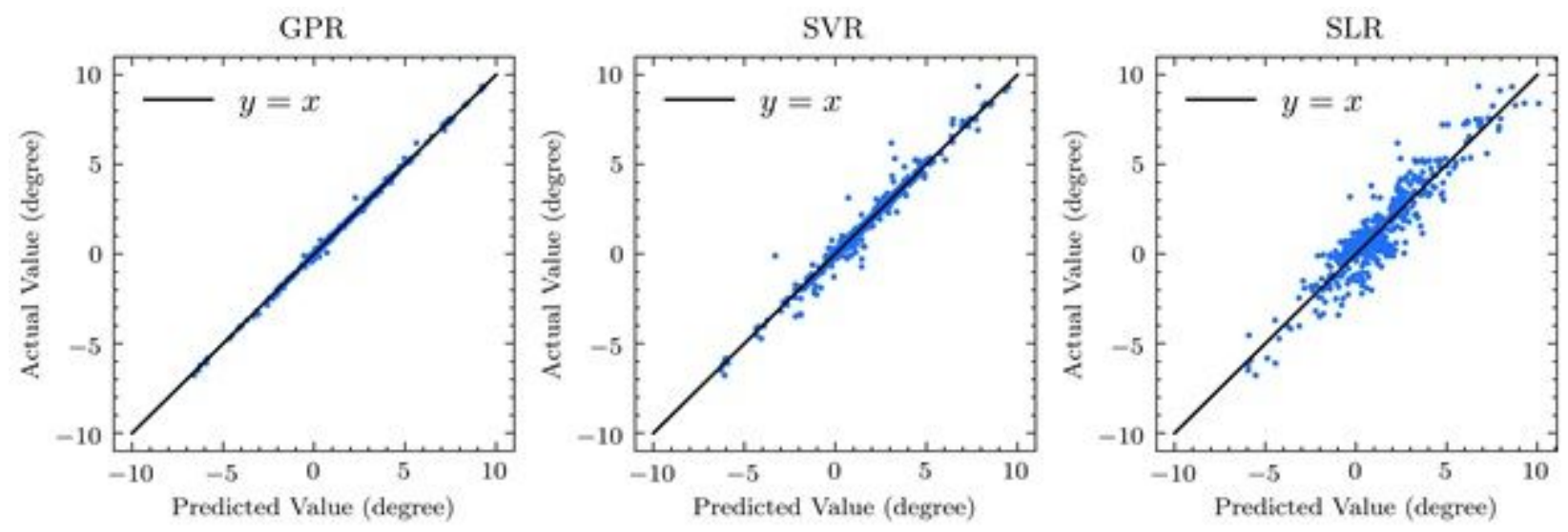

Figure 7

Actual angle and predicted angle of three regression models. 